\title{
PROPRIEDADES FÍSICAS DE UM LATOSSOLO VERMELHO DISTRÓFICO CULTIVADO E SOB MATA NATIVA(1)
}

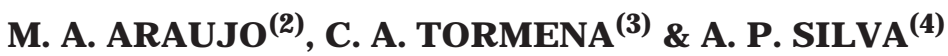

\begin{abstract}
RESUMO
A compreensão e a quantificação do impacto do uso e manejo na qualidade física dos solos são fundamentais no desenvolvimento de sistemas agrícolas sustentáveis. $\mathbf{O}$ objetivo deste trabalho foi avaliar algumas propriedades indicadoras da qualidade física de um Latossolo Vermelho distrófico da região Noroeste do Paraná, cultivado e sob mata nativa. Foram coletadas 24 amostras de solo com estrutura não deformada, na profundidade de 0-0,20 m, em duas áreas contíguas, sob mata nativa e cultivado, localizadas na Fazenda Experi mental da Universi dade Estadual de Maringá, município de Maringá, PR. A área cultivada tem sido utilizada com culturas anuais, com preparo convencional do solo (arado de discos e grade niveladora). A área sob mata nativa é classificada como Floresta Estacional semidecidual. Avaliaram-se a porosidade, a densidade do solo, a curva de retenção de água do solo, a curva de resistência do solo à penetração e o intervalo hídrico ótimo. Os resultados indicaram valores significativamente maiores de densidade do solo e menores de macroporosidade e porosidade total na área cultivada. Não foi constatado efeito significativo do uso do solo na curva de retenção de água, apesar de ter sido ela influenciada pela densidade do solo. A curva de resistência foi significativamente influenciada pelo uso do solo, evidenciada pelos maiores valores de resistência à penetração com o secamento do solo na área cultivada. O intervalo hídrico ótimo (IHO) foi menor no solo cultivado, uma vez que a resistência à penetração e a porosidade de aeração determinaram os limites, inferior e superior, de água di sponível com o aumento da densidade do solo. No solo sob mata nativa, o IHO foi igual à água disponível determinada pela capacidade de campo e pelo ponto de murcha permanente. A compactação do solo na área cultivada resultou em mudanças no sistema poroso, as quais foram descritas pelos menores valores do IHO na área cultivada.
\end{abstract}

Termos de indexação: degradação do solo, porosidade do solo, resistência do solo à penetração, intervalo hídrico óti mo.

(1) Parte da Tese de Mestrado do primeiro autor, apresentada no Curso de Pós-graduação em Agronomia da Universidade Estadual de Maringá - UEM. Recebido para publicação em setembro de 2002 e aprovado em novembro de 2003.

(2) Doutorando em Agronomia da Universidade Estadual de Maringá - UEM. Av. Colombo 5790, CEP 87020-900 Maringá (PR). Bolsista da CAPES. E-mail: araujomaa@yahoo.com.br

(3) Professor Adjunto do Departamento de Agronomia, UEM. Bolsista do CNPq. E-mail: catormena@uem.br

(4) Professor Associado da Escola Superior deAgronomia “Luiz de Queiroz" - ESALQ/USP. Caixa Postal 09, CEP 13418-900 Piracicaba (SP). Bolsista do CNPq. E-mail: apisilva@carpa.ciagri.usp.br 


\title{
SUMMARY: PHYSICAL PROPERTIES OF A DYSTROPHIC RED LATOSOL (OXISOL) UNDER CROP CULTIVATION AND NATIVE FOREST
}

\begin{abstract}
Theunderstanding and quantification of theimpact caused by soil useand management on thephysical soil quality arefundamental for the devel opment of sustainableagricultural systems. The objective of this research was to quantify some physical soil properties to evaluatethe physical quality of a dystrophic Red Latosol (RhodicF erralsol) in thenorthwest of theState of Paraná, Brazil. Undisturbed soil samples were coll ected from two contiguous areas on an Experimental Farm of theStateUniversity of M aringá. Onearea was cultivated with annual crops under conventional tillage (disk plow and harrowing) and the other under native forest (semidecidual seasonal forest). The evaluated physical soil attributes weresoil porosity and bulk density, soil water retention curve, soil resistancecurve, and the least limiting water range. Results indicated significantly higher values of bulk density and lower values of soil porosity in the cultivated area. The soil water retention curve was not influenced by the soil use system, but was negatively influenced by bulk density. The soil resistance curve was influenced by the soil use system, evidenced by high values of soil resistance to root penetration under increasing drought in the cultivated soil. The least limiting water range was significantly smaller in the cultivated soil owing to the soil resistanceto root penetration and air-filled porosity, which determined thelower and upper limits of soil water availability with bulk density increase. In the soil under nativeforest, the least limiting water range was equal to the water availability determined by the field capacity and the permanent wilting point. Soil compaction in the cultivated soil induced changes in the soil porous system, which weredescribed by lower values of theleast limiting water range than in virgin soil.
\end{abstract}

Index terms: soil degradation, soil porosity, soil resistance to penetration, least limiting water range

\section{INTRODUÇÃO}

A região NoroestedoParaná correspondea 17,6 \% da superfície do território estadual eapresenta solos derivados do arenito Caiuá, os quais representam $71,4 \%$ da área da região e, em sua maioria, são caracterizados por apresentarem textura superficial franco-arenosa e baixos teores de matéria orgânica (Carvalho, 1994). Devidoa fragilidade do ecossistema desta região, tem-se constatado a degradação física equímica dos sol os (Cardoso et al., 1992; Bragagnolo, 1994; F idalski, 1997). A degradaçãofísica destes sol os está relacionada com a redução nos teores dematéria orgânica (Cardoso et al., 1992), resultando em menor retenção de água e estabilidade dos agregados do solo (Costa \& Coel ho, 1990; Costa et al., 1997).

A compreensão e a quantificação do impacto do uso e manejo do solo na sua qualidade física são fundamentais no desenvolvimento de sistemas agrícolas sustentáveis (Dexter \& Y oungs, 1992). De acordo com Sanchez (1981), avaliações das modi ficações no solo decorrentes do cultivo deveriam ser feitas, submetendo um sol o sob vegetação natural às explorações agrícol as desejadas e analisando suas propriedades periodicamente. No entanto, por diferentes razões, é difícil atender a essas condições experimentais. Alternativamente, estes estudos podem ser feitos utilizando solos cultivados e sob mata nativa, desde que mantidos os critérios genéti cos etopográficos relacionados com a formação dos solos. No Brasil, têm sido feitos al guns estudos avaliando as mudanças nas propriedades dos solos utilizando o sol o sob mata como referência (Silva \& Ribeiro, 1992; Araújo et al., 1998; Dias J unior \& Estanislau, 1999; Sanches et al., 1999; Borges et al., 1999).

A introdução de sistemas agrícolas em substituição às florestas causa um desequilíbrio no ecossistema, modificando as propriedades do solo, cuja intensidade varia com as condições de clima, uso e manejos adotados e a natureza do solo (Godefroy \& J acquin, 1975). Com o uso intensivo dos sol os, geral mente ocorrea deterioração das suas propriedades físicas (Coote \& Ramsey, 1983). Modificações na densidade e na porosidade do solo podem variar consideravelmente, dependendo da textura, dos teores de matéria orgânica do solo (Curtis \& Post, 1964) e da freqüência de cultivo (Hajabbasi et al., 1997).

A qualidadefísica do sol o para o crescimento das plantas é determinada não só pela disponibilidade de água, aeração e temperatura, mas também pela resistência quea matriz do solo ofereceà penetração das raízes (Hamblin, 1985; Letey, 1985). Num solo degradado, além da redução da quantidade deágua disponível, a taxa de difusão de oxigênio e a resistência do solo à penetração podem limitar o crescimento das plantas na faixa de potenciais que determina a disponibilidade de água no solo. Desta forma, a caracterização dos efeitos dos sistemas de 
uso e manejo sobre a degradação e qualidade física do solo é mais bem quantificada por medidas integradoras destas modificações.

Neste contexto, o Intervalo Hídrico Ótimo (IHO), termo introduzido no Brasil por Tormena et al. (1998), a partir do trabalho de Silva et al. (1994), incorpora num único dado a amplitude de água do solo em que são mínimas as limitações ao desenvolvimento de plantas associadas à disponibilidade de água, aeração e resistência do solo à penetração. O IHO integra as propriedades físicas do solo que diretamente influenciam o desenvolvimento das culturas. Estas inter-relações dependem da condição estrutural do solo e, portanto, do grau de degradação da estrutura a que o sol o está submetido. Desta maneira, ol HO é utilizado como indicador da qualidade física e estrutural do solo, como proposto por Silva et al. (1994).

Os impactos do uso e manejo na qualidade física do solo têm sido quantificados, utilizando diferentes propriedades físicas relacionadas com a forma e com a estabilidadeestrutural do sol o, tais como: densidade do solo (De Maria et al., 1999; Stone \& Silveira, 2001), porosidade do solo (Beutler et al., 2001; Oliveira et al., 2001) eresistência dosol o à penetração das raízes (Tormena \& Roloff, 1996; De Maria et al., 1999; Rosolem et al., 1999; Beutler et al., 2001).

Neste sentido, Lal (1994) sugere que estas avaliações utilizem medidas integradoras do comportamento físico do solo, fazendo menção ao IHO. Os valores médios de propriedades, como densidade, água disponível e porosidade do solo, permitem comparar os efeitos dos sistemas de manejo e, por meio das suas magnitudes, inferir a respeito da qualidade física do solo. Por outrolado, a utilização do I HO permiteidentificar, por meio da quantificação e da integração dos dados relativos à água disponível, aeração e resistência do solo à penetração, as restrições impostas pela degradação estrutural à qualidade física do solo para o crescimento das plantas. I mhoff et al. (2001) utilizaram o conceito do IHO juntamente com a pressão de preconsol idação para estimar as pressões críticas que podem ser aplicadas sem causar a degradação da qualidade física do solo para o crescimento das plantas.

Neste contexto, o objetivo desta pesquisa foi avaliar a qualidadefísica de um L atossolo Vermel ho distrófico, cultivado e sob mata nativa, utilizando indicadores tradicionais, tais como: a densidade e a porosidade do solo e também indicadores não tradicionais como: a curva de resistência do solo à penetração e o IHO.

\section{MATERIAL E MÉTODOS}

Esteestudofoi realizadona F azenda Experimental da Universidade Estadual de Maringá, localizada no município de Maringá, região nor oeste do Paraná (L atitude $23^{\circ} 21^{\prime} \mathrm{S}$ e Longitude $52^{\circ} 03^{\prime} \mathrm{W}$ ). O solo utilizado é classificado como Latossolo Vermel ho distrófico (EMBRAPA, 1999). O material de origem deste solo provém de resíduos intemperizados do Arenito Caiuá da Série São Bento do período Cretáceo. N esta região, o tipo climático dominante é o Cfa na classificação de Köppen. O relevo é praticamente plano ou suave ondulado, com altitudes variando de $350-550$ m (EMBRAPA, 1984).

A amostragem foi realizada em maio de 1999. Foram selecionadas duas áreas próximas, uma sob mata nativa (Floresta estacional semidecidual) e outra cultivada por cerca de 20 anos, utilizando o sistema convencional de preparo do sol o com aração e gradagem, alternado com preparo mínimo do solo por meio de escarificação. Diferentes culturas têm sido utilizadas na área cultivada, sendo as mais comuns as de milho, aveia, sorgo, soja e mandioca. Por ocasião da amostragem, a cultura implantada era a da mandioca. E mambas as áreas, os resultados da análise granulométrica indicaram os seguintes teores de areia grossa $=350 \mathrm{~g} \mathrm{~kg}^{-1}$, de areia fina $=$ $410 \mathrm{~g} \mathrm{~kg}^{-1}$; desilte $=10 \mathrm{~g} \mathrm{~kg}^{-1}$ e deargila $=230 \mathrm{~g} \mathrm{~kg}^{-1}$, sendo sua classe textural franco-argilo-arenoso (EMBRAPA, 1999).

Em cada área foram selecionados, al eatoriamente, 24 pontos deamostrageme, em cada ponto, no centro da camada de 0-0,20 m de profundidade, coletou-se uma amostra com estrutura não deformada, utilizando amostrador com anel metálico de $50 \mathrm{~mm}$ de diâmetro e $50 \mathrm{~mm}$ dealtura, perfazendo um total de 48 amostras. Após a col eta, as amostras foram envoltas em papel alumínio e acondicionadas em sacos plásticos. No laboratório, as amostras foram preparadas para as análises, retirando-se o excesso de solo das suas extremidades. Em seguida, foram saturadas por meio da el evação gradual de uma lâmina de água numa bandeja, até atingir cerca de 2/3 da altura das amostras.

A porosidade total foi calculada como sendo o conteúdo de água do solo saturado. A quantificação dos valores de macroporosidade (Poros $\geq 50 \mu \mathrm{m}$ ) e microporosidade (Poros $<50 \mu \mathrm{m}$ ) foi obtida submetendo as amostras saturadas ao potencial de $-0,006 \mathrm{MPa}$ (EMBRAPA, 1997), utilizando uma mesa de tensão adaptada de Topp \& Zebtchuck (1979). Macropor os foram estimados como a diferença entre o conteúdo de água do sol o saturado e o conteúdo de água do solo após a aplicação do potencial de $0,006 \mathrm{MPa}$. O volume de microporos foi estimado como sendo o conteúdo de água retido no potencial de-0,006 MPa.

Para determinar a curva de retenção de água (CRA), foi adotado o procedimento descrito em Silva et al. (1994). As amostras foram divididas em 8 grupos de 6 amostras, sendo três amostras por sistema de uso do solo em cada potencial utilizado. Foram utilizados os seguintes potenciais: $-0,004,-0,006$, e 
-0,01 MPa numa mesa de tensão adaptada de Topp \& Zebtchuk (1979) e, -0,033, -0,07, -0,1, -0,3 e 1,5 MPa, utilizando pressões aplicadas em câmaras com placas porosas, conforme Klute (1986).

Cessada a drenagem e atingindo o equilíbrio hidráulico aparente, as amostras foram pesadas e, em seguida, foi determinada a resistência do solo à penetração, utilizando um penetrômetro, conforme método descrito por Tormena et al. (1998). Em seguida, as amostras foram secas em estufa a $\pm 105^{\circ} \mathrm{C}$, por $24 \mathrm{~h}$, para a determinação do conteúdo de água e da densidade do solo (Blake \& Hartge, 1986).

Para a determinação do IHO, é necessário quantificar as curvas de retenção de água e de resistência do solo à penetração. A curva de retenção de água no solo, expressa pela relação entre o potencial mátrico $(\psi)$ e o conteúdo de água $(\theta)$, foi estimada utilizando a função empregada por Ross et al. (1991), descrita na equação 1:

$$
\theta=a|\psi|^{b}
$$

na qual, aplicando-se a função logaritmo natural, chega-se à função linear descrita na equação 2:

$$
\ln \theta=\ln a+b \ln |\psi|
$$

em que $\theta=$ conteúdo vol umétrico de água $\left(\mathrm{m}^{3} \mathrm{~m}^{-3}\right)$, $\psi=$ potencial mátrico (MPa) e a e b coeficientes de ajuste da função. Os efeitos da densidade do solo (Ds) e do uso do solo (variável qualitativa: mata nativa $=0$, solo cultivado $=1$ ) foram avaliados conformeSilva et al. (1994). Desta forma, a equação 2 pode ser reescrita como:

$$
\begin{gathered}
\operatorname{In} \theta=\ln \left(a_{0}+a_{1} x_{1}+a_{2} x_{2}+a_{3} x_{1} x_{2}\right)+ \\
\left(b_{0}+b_{1} x_{1}+b_{2} x_{2}+b_{3} x_{1} x_{2}\right) \ln (|\psi|)
\end{gathered}
$$

em que $x_{1}, x_{2}$ ex $x_{1} x_{2}$ são, respectivamente, densidade do solo, uso do solo e a interação entre as duas variáveis, e $a_{i}$ e $b_{i}$ são os coeficientes determinados por regressão linear, utilizando o método dos mínimos quadrados.

A resistência do solo à penetração varia com o conteúdo de água e com a densidade do solo, e desta relação funcional pode-se determinar a curva de resistência do solo (CRS). A CRS foi ajustada por meio de um modelo não-linear, proposto por Busscher (1990), utilizando os procedimentos descritos por Silva et al. (1994). O model o utilizado é descrito na equação 4.

$$
R P=c \theta^{d} D s^{e}
$$

que, com a transformação logarítmica, resulta na equação 5:

$$
\text { In } \mathrm{RP}=\ln \mathrm{c}+\mathrm{d} \ln \theta+\mathrm{e} \text { In Ds }
$$

em queRP éa resistência do sol oà penetração( $\mathrm{MPa})$, $\theta$ é a umidade do solo $\left(\mathrm{m}^{3} \mathrm{~m}^{-3}\right)$ e Ds é a densidade do solo ( $\left.\mathrm{Mg} \mathrm{m}^{-3}\right)$, e c, d, e e são os coeficientes de ajuste do modelo. O procedimento para avaliar o efeito do uso do solo nos parâmetros do modelo (equação 5) foi semel hante ao adotado para a curva de retenção de água no solo (equação 3).

O IHO foi determinado com base nos procedimentos descritos por Tormena et al. (1998). Os valores críticos de umidade associados com o potencial mátrico, resistência do solo à penetração e porosidade de aeração foram, respectivamente: a capacidade de campo $\left(\theta_{\mathrm{CC}}\right)$ ou conteúdo de água estimado no potencial de -0,01 MPa (Reichardt, 1988); o ponto de murchamento permanente ( $\left.\theta_{\mathrm{PMP}}\right)$ ou conteúdo de água no potencial de -1,5 MPa (Savage et al., 1996); o conteúdo de água no sol o em que a resistência do solo à penetração $\left(\theta_{R P}\right)$ atinge 2,0 MPa (Taylor et al., 1966) e o conteúdo de água do solo em que a porosidade de aeração $\left(\theta_{\mathrm{PA}}\right)$ é de $0,10 \mathrm{~m}^{3} \mathrm{~m}^{-3}$ (Grable \& Siemer, 1968). Os valores de $\theta_{\mathrm{CC}}$ e $\theta_{\mathrm{PMP}}$ foram obtidos nos potenciais de -0,01 e -1,5 MPa, utilizando a curva de retenção de água, enquanto os val ores de $\theta_{\mathrm{RP}}$, em que ocorreresistência à penetração de 2,0 M Pa, foram obtidos por meio da curva de resistência do solo à penetração. $\mathrm{O}$ valor de $\theta_{\mathrm{PA}}$, em que a porosidade de aeração é de $0,10 \mathrm{~m}^{3} \mathrm{~m}^{-3}$, foi obtido por [(1-Ds/Dp)-0,1]. Considerou-se o val or de $2,65 \mathrm{Mg} \mathrm{m}^{-3}$ como sendo a densidade de partículas (Dp) do solo. O IHO foi calculado como a diferença entre olimite superior e inferior dos conteúdos de água: o limite superior é o menor valor de $\theta$ considerado na capacidade de campo ou na por osi dade de aeração de $10 \%$; olimite inferior é o maior valor de $\theta$ considerado, em que a resistência do solo à penetração atinge 2,0 $\mathrm{MPa}$ ou o valor de $\theta$ no ponto de murchamento permanente ( $\Psi=-1,5 \mathrm{MPa}$ ).

A comparação dos tratamentos para as variáveis densidade do solo, porosidade total, macro e microporosidade foi feita pelo testet para amostras independentes (Hatcher \& Stepanski, 1997). Os ajustes dos model os das curvas de retenção de água e de resistência do solo à penetração foram feitos com base na rotina PROC REG (SAS, 1999).

\section{RESULTADOS E DISCUSSÃO}

A Ds foi significativamente maior na área cul tivada em comparação com a área sob mata nativa (Figura 1).

Estes resultados estão de acordo com os obtidos por I slam \& Weil (2000), que constataram um valor médio da Ds significativamente maior em área cultivada comparada com sol o sob floresta natural. Também Silva \& Ribeiro (1992) obtiveram resultados similares, comparando sol o cultivado com cana e sob mata nativa. Sanches et al. (1999) constataram que, independentemente da posição de 


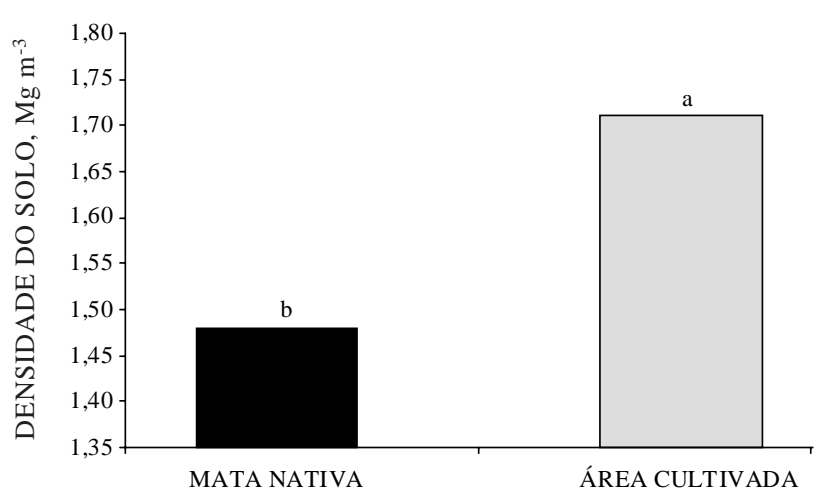

Figura 1. Valores médios de densidade do solo na área cultivada e sob mata nativa na profundidade de 0-0,20 m. Valores seguidos por letras minúsculas diferentes indicam diferenças significativas entre os tratamentos $(p<0,05)$.

amostragem, seja linha ou entrelinha da cultura da Iaranja, a Ds foi maior do queno sol o sob mata nativa.

A maior Ds nos solos cultivados está relacionada com a compactação do solo pel o tráfego de máquinas e implementos (Hajabbasi et al.,1997; Hartemink, 1998; Cavenage et al., 1999), com a redução dos teores de matéria orgânica (Silva \& Kay, 1997; Dalal $\&$ Chan, 2001) e com a menor estabilidade da estrutura do solo (Horn et al., 1995). O aumento da densidade do solo na área cultivada também pode ser explicado pela redução nos teores de matéria orgânica em comparação com o sol o sob mata nativa. Os teores de matéria orgânica foram de $18,4 \mathrm{~g} \mathrm{dm}^{-3}$, no solo sob mata nativa, e de $11,1 \mathrm{~g} \mathrm{dm}^{-3}$, no solo cultivado, estatisticamente diferentes pelo testet $(\mathrm{t}$ $=10,05 ; \mathrm{p}>\mathrm{t}=0,0001$ ).

Os valores de macroporos e de porosidade total do solo foram significativamente menores no solo cultivado em comparação com os do solo sob mata nativa (Figura 2). Resultados similares, em solo cultivado com cana-de-açúcar, foram obtidos por Silva \& Ribeiro (1992). A drástica redução da macroporosidade nos solos cultivados decorre do aumento da compactação do sol o, que é evidenciada pelo aumento da densidade do solo (Borges et al., 1999; Kay \& Angers, 1999).

A macropor osidade, ou a porosidade ocupada com ar no potencial de $-0,006 \mathrm{MPa}$, é uma medida relacionada com a taxa de difusão de oxigênio no solo. Os resultados obtidos por Thomasson (1978) em sol os temperados indicam quea rápi da drenagem do sol o cessa em potenciais da ordem de -0,005 $\mathrm{MPa}$ e os poros drenados neste potencial determinam a capaci dade de aeração dos solos. Utilizando o critério estabelecido por Thomasson (1978) e os valores médios de macroporosidade obtidos neste estudo (Figura 2), constatou-se quea capacidade de aeração do solo na área cultivada é classificada como inadequada, enquanto no solo sob mata é considerada muito boa. Salienta-se que valores adequados de capacidade de aeração são dependentes das condi ções climáticas e devem ser ampliados sob condições mais úmidas.

$\mathrm{Na}$ microporosidade do solo, não foi constatada diferença significativa $(p>0,05)$ entre os dois sistemas de uso avaliados. Silva \& Kay (1997) salientaram que a microporosidade do solo é fortemente influenciada pela textura, teor de carbono orgânico e muito pouco influenciada pelo aumento da densidade do solo, originada do tráfego de máquinas, implementos, etc.

As CRS, obtidas a partir do ajuste dos dados da equação 4, são descritas nas equações 6 e 7 . Todos os coeficientes do ajuste são estatisticamente significativos $(p<0,05)$. A RP é positivamente correlacionada com a Ds e negativamente $\operatorname{com} \theta$ em ambos os sistemas de uso avaliados, corroborando os resultados obtidos por Silva et al. (1994), Tormena et al. (1998) e I mhoff et al. (2001). Os resultados indicaram um aumento significativamente maior da resistência do sol o à penetração com a perda deágua no solo cultivado comparado ao sol o sob mata nativa $(p<0,05)$. O modelo ajustado explicou $84 \%$ da variabilidade dos dados de resistência do solo à penetração.

$$
\begin{aligned}
& \text { Mata nativa: } \quad R P=0,0132574 D s^{5,3229} \theta^{-1,3406} \\
& F=57,33 ; p=0,0001 ; R^{2}=0,84 ; N=24
\end{aligned}
$$

Área cultivada: $\mathrm{RP}=0,0028515 \mathrm{Ds}^{5,3229} \theta^{-2,0592}$

$$
\begin{aligned}
& F=57,33 ; p=0,0001 ; R^{2}=0,84 ; \\
& N=24
\end{aligned}
$$

A representação gráfica da RP em função de Ds e $\theta$ é ilustrada nas figuras 3 e 4, respectivamente, para o sol o sob mata nativa e cultivado.

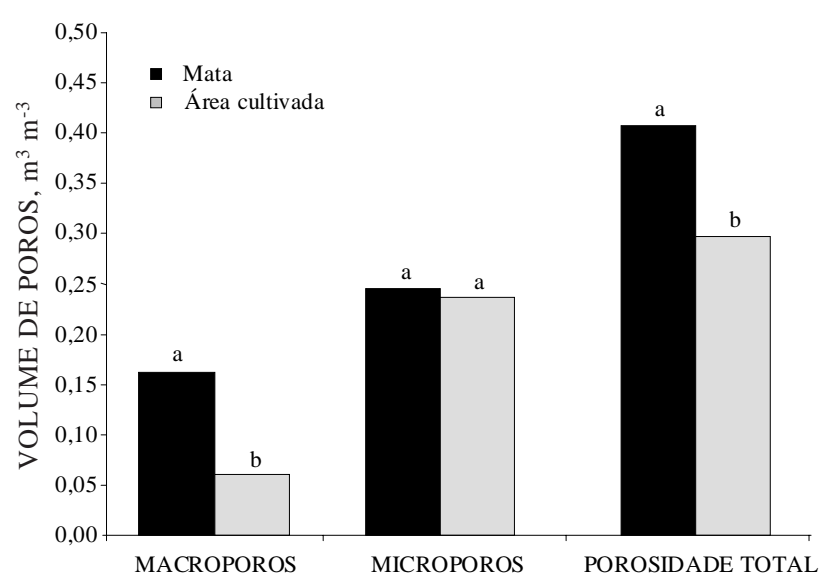

Figura 2. Valores médios de macro, micro e porosi dade total do solo na área cultivada e sob mata nativa na profundidade de $0-0,20 \mathrm{~m}$. Valores seguidos por letras minúsculas diferentes indicam diferenças significativas entre os tratamentos $(p<0,05)$. 


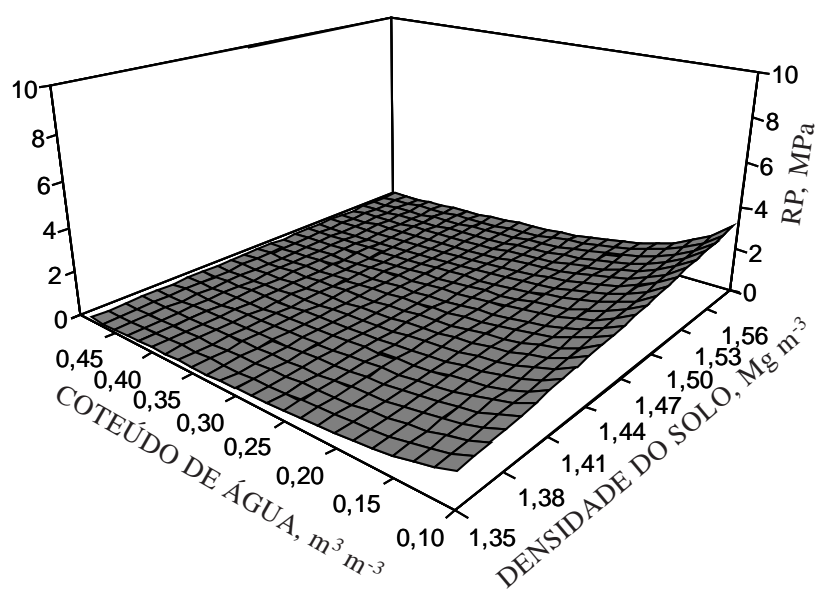

Figura 3. Curva de resi stência à penetração do solo sob mata nativa.

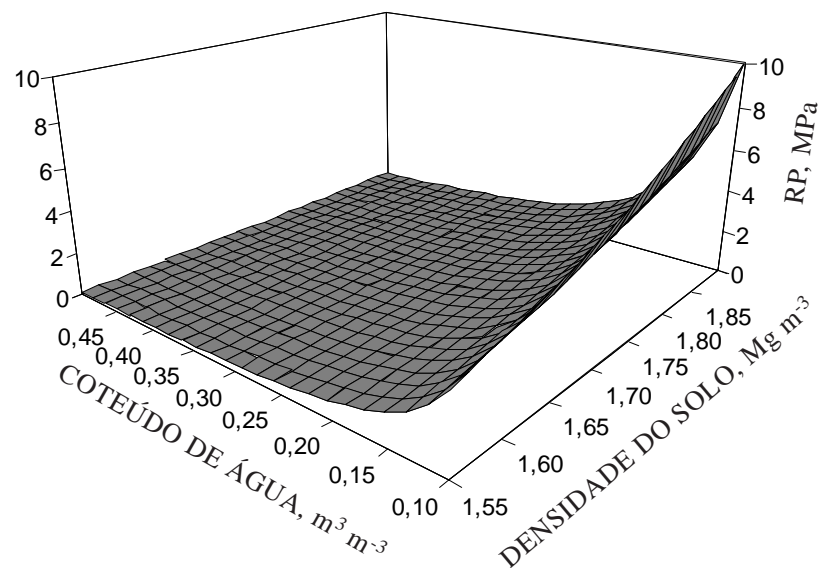

Figura 4. Curva de resistência à penetração do solo cultivado.

Com o secamento, o solo cultivado apresentou valores de RP mais altos do que o solo sob mata nativa (Figuras 3 e 4). No solo sob mata, os valores de RP atingem níveis considerados críticos ao crescimento das raízes ( $\mathrm{RP} \geq 2,0 \mathrm{MPa}$ ) somente para conteúdos de água muito baixos e em valores de Ds el evados. Comparando as figuras 3 e 4, constata-se que o maior val or de Ds encontrado no sol o sob mata nativa é muito próximo do menor valor de Ds encontrado no sol o sob cultivo e, nestes dois pontos, os valores da RP, no mesmo conteúdo de água do solo, são muito semelhantes, indicando que os mai ores valores de densidade do solo na área cultivada causaram mai or sensibilidade da resistência do solo à penetração em relação à umidade, o que foi constatado pelo coeficiente significativamente maior no solo cultivado. Estes resultados estão em concordância com os de Murphy et al. (1995). O aumento dos valores de RP com o decréscimo da $\theta$ pode estar associado com o aumento do "stress efetivo" (Giarola et al., 2003) ou com a maior coesão entre as partículas minerais (Kay \& Angers, 1999), sendo maior no sol o cultivado.
Não foi constatado efeito significativo do uso do solo nos parâmetros da equação 1, utilizada para ajustar os dados da CRA. Por outrolado, constatouse quea Ds influenciou significativamentee de forma negativa a retenção de água $(p<0,05)$. A Ds tem ação sobre a retenção de água, decorrente de sua influência na porosidade total e distribuição do tamanho dos poros (Archer \& Smith, 1972). Cerca de $78 \%$ da variabilidade da retenção deágua no sol o foi explicada pelo modelo ajustado aos dados (equação 8).

A determinação do IHO exige estimativas dos conteúdos de água na capacidade de campo (CC, potencial de -0,01 MPa) e no ponto de murcha permanente (PMP, potencial de -1,5 MPa). A equação utilizada para esta finalidade, tanto para mata nativa quanto para área cultivada, é descrita pela equação 8.

$$
\begin{gathered}
\theta=\left(e^{(-1,1036-0,3635 D s)}\right) *\left(|\psi|^{-0,1535}\right) \\
F=80,68 ; p=0,0001 ; \\
R^{2}=0,78 ; N=48
\end{gathered}
$$

As estimativas do conteúdo de água em quea RP atingiu 2,0 MPa foram obtidas das equações 6 e 7, as quais são apresentadas nas equações 9 e10, para as áreas avaliadas.

Mata nativa: $\quad \theta=\left(\mathrm{RP} /\left(0,0132574 \mathrm{Ds}^{5,3229}\right)\right)^{1 /-1,3406}(9)$

Área cultivada: $\theta=\left(\mathrm{RP} /\left(0,0028515 \mathrm{Ds}^{5,3229}\right)\right)^{1 /-2,0592}(10)$

Os conteúdos deágua nos limites críticos do I HO, no sol o sob mata nativa e cultivado, são apresentados nas figuras 5 e 6, respectivamente.

Em ambos os sistemas de uso, com o aumento na Ds, ocorreram simultaneamente um aumento na $\theta_{\mathrm{RP}}$ e um decréscimo na $\theta_{\mathrm{PA}}$ (Figuras 5 e 6$)$, concordando com os resultados obtidos por Silva et al. (1994), Tormena et al. (1998) el mhoff et al. (2001). No solo sob mata nativa, os resultados indicam que a maior disponibilidade de água ocorreu entrea CC eoPMP, exceto para duas amostras com valores maiores de Ds na distribuição (Figura 5), nos quais os valores de $\theta_{\mathrm{PMP}}$ e $\theta_{\mathrm{RP}}$ foram praticamente iguais. Na área sob mata nativa, em praticamente toda a variação de Ds, ol HO teve como limite superior e inferior os val ores de umidade correspondentes a CC eao PMP. Estes resultados evidenciam que, na área sob mata nativa, as limitações físicas ao crescimento das plantas são determinadas pel os potenciais relativos a CC e PMP ao longo de praticamente toda faixa de Ds avaliada, corroborando as indicações de que solos sob mata nativa apresentam excelentes condições físicas (Reynolds et al., 2002).

No solo cultivado, a $\theta$ na qual a porosidade de aeração $\leq 10 \%\left(\theta_{\mathrm{PA}}\right)$ foi menor do que a CC a partir da densidade de $1,71 \mathrm{Mg} \mathrm{m}^{-3}$ (Figura 6), ou seja, a partir deste valor de densi dade, quando o solo estiver na capacidade de campo, a difusão de oxigênio pode 


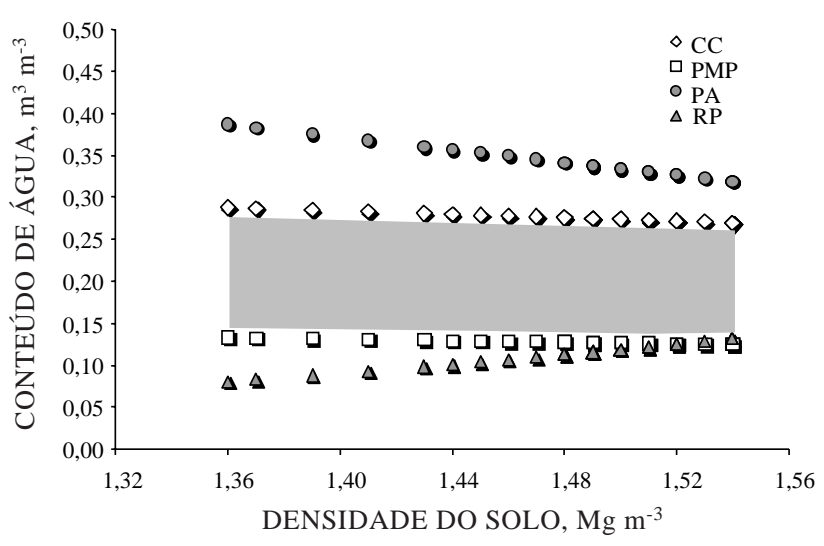

Figura 5. Variação do conteúdo de água com a densidade, no solo sob mata nativa, nos níveis críticos de capacidade de campo (CC), ponto de murcha permanente (PMP), porosidade de aeração de $10 \%$ (PA) e resi stência à penetração de 2,0 MPa (RP). A área hachurada representa o Intervalo Hídrico Ótimo do Solo (I HO).

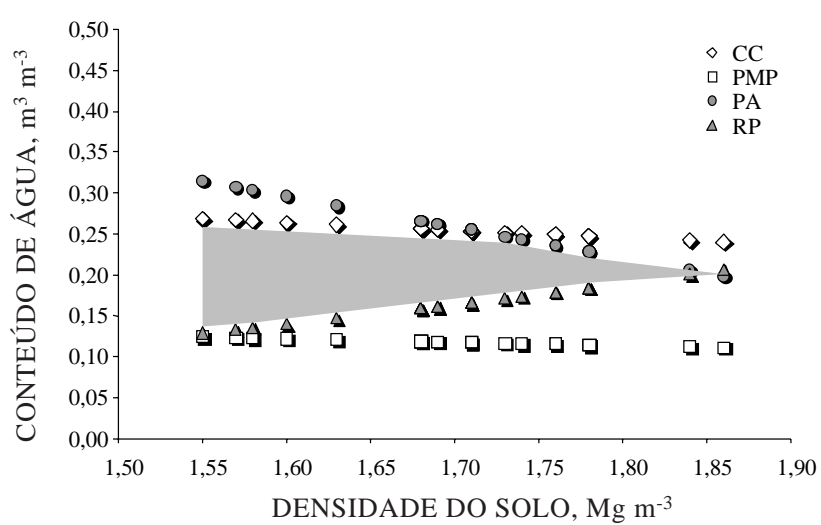

Figura 6. Variação do conteúdo de água com a densidade, na área sob cultivos anuais, nos níveis críticos de capacidade de campo (CC), ponto de murcha permanente (PMP), porosidade de aeração de $10 \%$ (PA) e resistência à penetração de 2,0 $\mathrm{MPa}$ (RP). A área hachurada representa o I ntervalo Hídrico Ótimo do Solo (IHO).

ser insatisfatória, acarretando condições físicas desfavoráveis para o crescimento das culturas.

No sol o cultivado, a variação da Ds teve também grande impacto sobre a RP. A $\theta_{\text {RP }}$ substituiu a $\theta_{\text {PMP }}$ nos valores de $\mathrm{Ds} \geq 1,55 \mathrm{Mg} \mathrm{m}^{-3}$ (Figura 6). Estes resultados estão de acordo com os obtidos por Topp et al. (1994), Silva et al . (1994), Tormena et al . (1998; 1999) e Imhoff et al. (2001), nos quais a RP foi o fator que mais freqüentemente reduziu o IHO em sol os sob diferentes condições de textura e manejo. $\mathrm{Na}$ literatura, há exemplos da ocorrência de impedimentos ao crescimento das plantas pela RP sob potenciais maiores que o PMP (Boone et al., 1986).
No sol o sob mata nativa, o IHO foi igual à água disponível e, no solo cultivado, com o aumento da Ds, constatou-se redução no I HO decorrente da atuação simultânea da porosidade de aeração e da RP. De acordo com Kay (1989), o decréscimo dol HO caracteriza a perda da qualidade física do solo, considerando a maior probabilidade de as culturas serem expostas a estresses físicos, o que foi constatado por Silva \& Kay (1996). Estes resultados evidenciam que, comparativamente ao sol o sob mata nativa, a degradação da estrutura do sol o pel o cultivo impõe limitações ao crescimento das plantas pela redução na água disponível e pela restrição de aeração sob el evada umidade do sol o ou pela el evação da resistência do sol oà penetração, que pode atingir valores altos, ao longo do secamento do solo.

\section{CONCLUSÕES}

1. O solo sob cultivo apresentou maiores valores de densidade do sol o emenores valores de porosi dade total e de macroporosidade comparado com o solo sob mata nativa.

2. A resistência do solo à penetração foi influenciada positivamente pela densidade e negativamente pela umidade do solo, com maior magnitude no solo cultivado. A densidade do solo afetou a retenção de água, refletindo os efeitos do uso do solo.

3. No solo sob mata nativa, ol HO foi igual ao da água disponível. No sol o cultivado, o IHO foi menor do que no solo sob mata nativa em decorrência da influência da porosidade de aeração e da resistência do solo à penetração que estabeleceram os limites de disponibilidade de água no solo.

\section{LITERATURA CITADA}

ARAUJ O, Q.R.; COSTA, L.M.; J UCKSCH, I.; FONTES, L.E.F. \& REGAZZI, A.J. Alterações nas propriedades físicas de um podzólico vermelho-amarelo da região cacaueira da Bahia, sob diferentes coberturas vegetais. Agrotrópica, 10:69-78, 1998.

ARCHER, J.R. \& SMITH, P.D. The relation between bulk density, available water capacity and air capacity of soils. J . Soil Sci., 23:475-480, 1972.

BEUTLER, A.N.; SILVA, M.L.N.; CURI, N.; FERREIRA, M.M.; CRUZ, J.C. \& PEREIRA FILHO, I.A. Resistência à penetração e permeabilidade de Latossolo Vermel ho distrófico típico sob sistemas de manejo na região dos cerrados. R. Bras. Ci. Solo, 25:167-177, 2001.

BLAKE, G.R. \& HARTGE, K.H. Bulk density. In: KLUTE, A., ed. Methods of soil analysis: physical and mineralogical methods. 2.ed. Madison, America Society of Agronomy, 1986. p.363-375. 
BOONE, F.R.; van der WERF, H.M.G.; KROESBERGEN, B.; TEN HAAG, B.A. \& BOERS, A. The effect of compaction of arable layer in a sandy soils on the growth of maize for silage. I. Critical potentials in relation to soil aeration and mechanical impedance. Neth. J. Agric. Res., 34:155-171, 1986.

BORGES, A.L.; KIEHL, J.C. \& SOUZA, L.S. Alteração de propriedades físicas eatividade microbiana de um latossolo amarelo álico após o cultivo com fruteiras perenes e mandioca. R. Bras. Ci. Solo, 23:1019-1025, 1999.

BRAGAGNOLO, N. Uso dos solos altamente susceptíveis à erosão. In: PEREIRA, P.P.; FERREIRA, M.E. \& PESSOA DA CRUZ, M.C., eds. Sol os altamente susceptíveis à erosão. J aboticabal, FCAV-UNESP/SBCS. 1994. p.3-16.

BUSSCHER, W.J . Adjustment of flat-tipped penetrometer resistance data to common water content. Trans. Am. Soc. Agric. Eng., 3:519-524, 1990.

CARDOSO, A.; POTTER, R. \& DEDECEK, R.A. Estudo comparativo da degradação de solos pelo uso agrícola no Noroeste do estado do Paraná. Pesq. Agropec. Bras., 27:349353, 1992.

CARVALHO, A.P. Solos do arenito caiuá. In: PEREIRA, P.P.; FERREIRA, M.E. \& PESSOA DA CRUZ, M.C., eds. Solos altamente susceptíveis à erosão. J aboticabal, FCAVUNESP/SBCS, 1994. p.39-49.

CAVENAGE, A.; MORAES, M.L.T.; ALVES, M.C.; CARVALHO, M.A.C.; FREITAS, M.L.M. \& BUZETTI, S. Alterações nas propriedades físicas de um latossolo vermel ho-escuro sob diferentes culturas. R. Bras. Ci. Solo, 23:997-1003, 1999.

COOTE, D.R. \& RAMSEY, J .F. Quantification of the effects of overs 35 years of intensive cultivation on four soils. Can. J . Soil Sci., 63: 1-14, 1983.

COSTA, A.C.S. \& COELHO, S.M.R. Efeito do manejo do solo em Latossolo Vermelho-Escuro textura média-LEd2 do Município de Paranavaí-Paraná.II. Estabilidade dos agregados em água. In: CONGRESSO BRASILEIRO E ENCONTRO NACIONAL DE PESQUISA SOBRE CONSERVAÇÃO DE SOLOS, 8., Londrina, 1990. Resumos. Londrina, 1990. p.35.

COSTA, A.C.S.; NANNI, M.R.\& J ESKE, E. Determinação da umidade na capacidade de campo e ponto de murcha permanente por diferentes metodologias. R. Unimar, 19:827-844, 1997.

CURTIS, R.O. \& POST, B.W. Estimating bulk density from organic matter content in some Vermont forest soils. Soil Sci. Soc. Am. Proc., 28:285-286, 1964.

DALAL, R.C. \& CHAN, K.Y. Soil organic matter in rainfed cropping systems of the Australian cereal belt. Aust. J . Soil Res., 39:435-464, 2001.

DE MARIA, I.C.; CASTRO, O.M. \& SOUZA DIAS, H. Atributos físicos do solo e crescimento radicular de soja em Latossolo Roxo sob diferentes métodos de preparo do solo. R. Bras. Ci. Solo, 23:703-709, 1999.

DEXTER, A.R. \& YOUNGS, I.M. Soil physic toward 2000. Soil Till. Res., 24:101-106, 1992.
DIAS JUNIOR, M.S. \& ESTANISLAU, W.T. Grau de compactação e retenção de água de latossolos submetidos a diferentes sistemas de manejo. R. Bras. Ci. Solo, 23:4551, 1999.

EMPRESA BRASILEIRA DE PESQUISA AGROPECUÁRIA EMBRAPA.. Levantamento de reconhecimento dos solos do estado do Paraná. Curitiba, 1984. (Boletim técnico 57)

EMPRESA BRASILEIRA DE PESQUISA AGROPECUÁRIA EMBRAPA. Manual de métodos de análises de solos. 2.ed. Rio de J aneiro, 1997. 212p.

EMPRESA BRASILEIRA DE PESQUISA AGROPECUÁRIA EMBRAPA. Sistema Brasileiro de Classificação de Solos. 1999. $412 p$.

FIDALSKI,J . Fertilidade do sol o sob pastagens, lavouras anuais e permanentes na região noroeste do Paraná. R. Unimar, 19:853-861, 1997.

GIAROLA, N.F.B.; SILVA, A. P.; IMHOFF, S. \& DEXTER, A.R. Contribution of natural soil compaction on hardsetting behavior. Geoderma, 113:95-108, 2003.

GODEFROY, J . \& J ACQUIN, F. Relation entre la stabilité structurale des sols cultivés et le apports organiques en conditions tropicales;comparasion avec les sols forestiers. Fruits, 30:595-612, 1975

GRABLE, A.R. \& SIEMER, E.G. Effects of bulk density, aggregate size, and soil water suction on oxygen diffusion, redox potential and elongation of corn roots. Soil Sci. Soc. Am. J ., 32:180-186, 1968.

HAJ ABBASI, M.A.; J ALALIAN, A. \& KARIMZADEH, H.R. Deforestation effects on soil physical and chemical properties, Lordegan, Iran. Plant Soil, 190:301-308, 1997.

HAMBLIN, A.P. The influence of soil structure on water movement, crop root growth and water uptake. Adv. Agron., 38:95-158, 1985.

HARTEMINK, A.E. Soil chemical and physical properties as indicators of sustainable land management under sugar cane in Papua New Guinea. Geoderma, 85:283-306, 1998.

HATCHER, L. \& STEPANSKI, E.J . A step-by-step approach to using the SAS System for Univariate and Multivariate Statistics. Cary, SAS Institute, 1997. 552p.

HORN, R.; DOMZAL, H.; SLOWINSKA-J URKIEWICZ, A. \& van OUWERKERK, C. Soil compaction processes and their effects on the structure of arable soils and the enviroment. Soil Till. Res., 35:23-36, 1995.

IMHOFF, S.; DA SILVA, A.P.; DIAS J ÚNIOR, M.S.\& TORMENA, C.A. Quantificação de pressões críticas para o crescimento de plantas. R. Bras. Ci. Solo, 25:11-18, 2001.

ISLAM, K.R. \& WEIL, R.R. Land use effects on soil quality in a tropical forest ecosystem of Bangladesh. Agric. Ecosys. Environ., 79:9-19, 2000.

KAY, B.D. Rates of changes of soil structure under different cropping systems. Adv. Soil Sci., 12:1-51, 1989.

KAY, B.D. \& ANGERS, D.A. Soil structure. In: A. SUMNER, M.E., ed. Handbook of Soil Science. Boca Raton, CRC Press, 1999. p.229-276 
KLUTE, A. Water retention: Laboratory Methods. In: KLUTE, A., ed. Methods of soil analysis - physical and mineralogical methods. Madison, America Society of Agronomy, 1986. p.635-660.

$L A L, R$. Methods and guidelines for assessing sustainable use of soil and water resources in the tropics. Soil Management Support Service, USDA, U.S. Agency for International Development. Ohio, Ohio State University, 1994. 78p. (Technical Monograph $n^{\circ} 21$ )

LETEY, J. Relationship between soil physical properties and crop production. Adv. Soil Sci., 1:277-294, 1985.

MURPHY, W.M.; BARRETO, A.D.; SILMAN, J .P. \& DINDAL, D.L. Cattle and sheep grazing effects on soil organisms, fertility and compaction in a smooth-stal ked meadowgrassdominant white clover sward. Grass Forrage Sci., 50:191194, 1995.

OLIVEIRA, J .O.A.P.; VIDIGAL FILHO, P.S.; TORMENA, C.A.; PEQUENO, M.G.; SCAPIM, C.A.; MUNIZ, A.S. \& SAGRILO, E. I nfluência de sistemas de preparo do sol o na produtividade da mandioca (Manihot esculenta, Crantz). R. Bras. Ci. Solo, 25:443-450, 2001.

REICHARDT, K. Capacidade decampo. R. Bras. Ci. Solo, 12:211216, 1988.

REYNOLDS, W.D.; BOWMAN, B.T.; DRURY, C.F.; TAN, C.S. $\& L U, X$. Indicators of good soil physical quality: density and storage parameters. Geoderma, 110:131-146, 2002.

ROSOLEM, C.A.; FERNANDEZ, E.M.; ANDREOTTI; M. \& CRUSCIOL, C.A.C. Crescimento radicular de plântulas de milho afetado pela resistência do solo à penetração. Pesq. Agropec. Bras., 34:821-828, 1999.

ROSS, P.J .; WILLIANS, J . \& BRISTOW, K.L. Equations for extending water-retention curves to drynees. Soil Sci. Soc. Am. J ., 55:923-927, 1991.

SANCHES, A.C.; SILVA, A.P.; TORMENA, C.A. \& RIGOLIN A.T. I mpacto do cultivo de citros em propriedades químicas, densidade do solo e atividade microbiana de um Podzólico Vermelho-Amarelo. R. Bras. Ci. Solo, 23:91-99, 1999.

SANCHEZ, P.A. Suelos del trópico - características y manejo. San J osé, I nstituto I nteramericano de Cooperacion para la Agricultura, 1981. 645p.

SAVAGE, M.J .; RITCHIE, J .T.; BLAND, W.L. \& DUGAS, W.A. Lower limit of soil water availability. Agron. J ., 88:844851, 1996.
SILVA, A.P. \& KAY, B.D. The sensitivity of shoot growth of corn to the least limiting water range of soils. Plant Soil, 184:323-329, 1996.

SILVA, A.P. \& KAY, B.D. Estimating the least limiting water range of soils from properties and management. Soil Sci. Soc. Am. J ., 61:877-883, 1997.

SILVA, A.P.; KAY, B.D. \& PERFECT, E. Characterization of the least limiting water range. Soil Sci. Soc. Am. J ., 58:17751781, 1994

SILVA, M.S.L. \& RIBEIRO, M.R. Influência do cultivo contínuo da cana-de-açúcar em propriedades morfológicas e físicas de solos argilosos de tabuleiro no estado de Alagoas. R. Bras. Ci. Solo, 16:397-402, 1992.

SAS INSTITUTE. Statistical Analysis System. Procedure guide for personal computers. Cary, 1999.

STONE, L.F. \& SILVEIRA, P.M. Efeitos do sistema de preparo e da rotação de culturas na porosidade e densidade do solo. R. Bras. Ci. Solo, 25:395-401, 2001.

TAYLOR, H.M.; ROBERSON, G.M. \& PARKER J r., J J . Soil strength-root penetration relations to medium to coarsetextured soil materials. Soil Sci., 102:18-22, 1966.

THOMASSON, A.J. Towards objective classification of soil structure. J. Soil Sci., 29:38-46, 1978.

TOPP, G.C.; GALGANOV,Y.T.; WIRES, K.C. \& CULLEY, J .L.B. Non limiting water range (NLWR): an approach for assessing soil structure. Soil Quality Evaluation Program. Agriculture and Agri-Food Canada, 1994. 36p. (Technical report, 2)

TOPP, G.C. \& ZEBTCHUK, W. The determination of soil water desorption curves for soil cores. Can. J . Soil Sci., 59:19-26, 1979.

TORMENA, C.A.; DA SILVA, A.P. \& LIBARDI, P.L. Soil physical quality of a Brazilian Oxisol under twotillagesystems using the least limiting water range approach. Soil Till. Res., 52:223-232, 1999.

TORMENA, C.A.; SILVA, A.P. \& LIBARDI, P.L. Caracterização do intervalo hídrico ótimo de um $L$ atossolo Roxo sob plantio direto. R. Bras. Ci. Solo, 22:573-581, 1998.

TORMENA, C.A. \& ROLLOF, G. Dinâmica da resistência à penetração de um solo sob plantio direto. R. Bras. Ci. Solo, 20:333-339, 1996. 
M.A. ARAUJ O et al.

R. Bras. Ci. Solo, 28:337-345, 2004 